\title{
Methodisches Denken im Poststrukturalismus
}

Ist humangeographisches Wissen im angelsächsischen Sprachraum oft durch eine klar erkennbare Periodizität und also durch eine gewisse Anfälligkeit gegenüber Modeerscheinungen gekennzeichnet, so ist dessen deutschsprachiges Pendant besser durch die Tendenz zur Schulbildung charakterisiert. Da letztere mehr Zeit beansprucht, überrascht es nicht, daß die Übernahme oder Rezeption von Ideen im deutschsprachigen Umfeld zwar genau, aber oft auch spät stattfindet. Dies trifft in besonderem Maße auf das Themenumfeld «Poststrukturalismus» zu, weshalb ich hier zunächst einige Schlüsselbegriffe erläutern möchte.

Da steht es an, zunächst den Kontext zu bedenken. Die wichtigste Umwälzung in den Sozial- und Humanwissenschaften der vergangenen drei Jahrzehnte war sicherlich durch den graduellen Einbezug von «Sprache» in die Begriffs- und Problemregister der jeweiligen Wissenschaften, den sogenannten "linguistic turn», markiert. Nicht zufällig umschreibt der genannte Zeitraum dabei die Zeitspanne, die seit Mai 1968 vergangen ist: die Reflexion über Sprache ging Hand in Hand mit der nach den sogenannten Studentenunruhen eher schrittweise einsetzenden Ernüchterung angesichts der weitgehenden Ineffektivität politischer wie wissenschaftlicher Diskurse. In der Folge wurden nicht allein die Begriffe «Text» und «Diskurs» neu bedacht, darüber hinaus wurde auch die von LYOTARD (1979) vorgetragene Kritik sogenannter «grands récits» oder hegemonialer Begriffswelten (à la «Fortschritt», «Freiheit» oder «Emanzipation») unter dem Oberbegriff der «Postmoderne» zunehmend salonfähig. Gemäß dem wissenschaftstheoretischen Teil dieser Kritik, welcher seit Mitte der 1980er Jahre auch Humangeographen' überzeugt hat, war der Wissenschaft vermittels ihrer sprachlichen Konstitution ein willkürliches Element zu eigen, welches zugrundeliegende Machtansprüche oft nur durch Idealisierungen rational zu verkleiden wußte. Hier wurde also die Frage nach der Rationalität der Vernunft und der aus ihr sich ergebenden Legitimation von Moderne - oft im unbewußten Anschluß an NIETZSCHE? - abermals gestellt. Diese Gedankengänge wurden in der Geographie ab den späten 1980er Jahren gestellt und können im Folgenden als bekannt vorausgesetzt werden (kritisch dazu vor allem BECKER 1990 und HARVEY 1989; wohlwollend DEAR 1988, 1994 und SOJA 1989).

Weitestgehend ungeklärt blieb dabei in der sich ergebenden Diskussion, ob «Sprache» im weitesten Sinne als ermöglichend oder als verhindernd verstanden werden muß. Der bewußte Einbezug von «Sprache» in die Sozialwissenschaften, das Bedenken des Mediums wissenschaftlicher Kommunikation, ist ja an und für sich noch unbedingt. Er schließt weder eine pragmatisch oder kreativ erschließende noch eine kritisch ausschließende Interpretation von "Sprache» aus. Ist «Sprache» also funktionierendes Medium oder aber verzögerndes, wenn nicht ausschließendes Hindernis auf dem Weg zur wissenschaftlichen Erkenntnis? Von der individuellen (und oft impliziten) Beantwortung dieser Frage hing dann auch ab, welche Schlußfolgerungen im einzelnen aus der «postmodernen» Kritik gezogen wurden. Auf der einen Seite wurde optimistisch «Sprache» als alternativlose, wenn auch stets kritisierbare Annäherung an eine zu erklärende Wirklichkeit verstanden und so dem willkürlichen Element sprachlicher Bestimmungen ein selbstregulierendes Moment zur Seite gestellt (so auch bei HARD 1990); andererseits fanden sich Vertreter einer pessimistischen Einschätzung genötigt, die postmoderne Einschätzung zur «poststrukturalistischen» Kritik zu radikalisieren. Letztere verstand Sprache als nicht zu hintergehenden Grund einer jeden Form von Kommunikation, deren strukturelle Eigenarten somit auch prägend für wissenschaftliche Erkenntnis sind (DERRIDA 1967). Als entscheidend erwies sich hierbei, daß die schon von SAUSSURE benannte grundsätzliche Zufälligkeit einer jeden Benennung (oder eines jeden Namens) sich auch in wissenschaftlichen Diskursen nicht rational in etwas Bestimmtes, Eindeutiges überführen läßt, sondern allenfalls durch eine ihrerseits nicht wiederum zu begründende Schaffung von Eindeutigkeiten verdrängt werden kann. Laut dieser Lesart ist also das Ziel wissenschaftlichen Tuns, weil es unhintergehbar ein sprachliches Tun ist und bleiben wird, nicht mit wissenschaftlich-rationalen Mitteln allein einlösbar, sondern bedarf, in Anlehnung an NIETZSCHE, eines potentiell stets irrationalen Willens zur Wissenschaft. Solcherart ihres modernen Selbstverständnisses beraubt, bleiben der Wissenschaft allein die permanente Rückbesinnung auf die eigenen Selbstverständlichkeiten ${ }^{2}$ und deren Kritik als «wissenschaftlicher» Weg offen, so zumindest bei der im Gedanken der «Dekonstruktion» mündenden, poststrukturellen Variante Jacques DERRIDAS. «Post-»strukturell sind diese Gedanken also gleich in mehrerer Hinsicht:

Ulf Strohmayer, Dr., Reader, Department of Geography, University of Wales, Lampeter 
sie verabschieden den Glauben an die Erkennbarkeit von Strukturen durch dessen Aufdeckung als sprachlich formulierten Glauben; sie anerkennen das Vorhandensein des Erkenntnisversuches "Wissenschaft» als nicht zu hintergehenden Versuch; und sie beharren auf der Notwendigkeit der Fortschreibung des sozialen Erkenntnisprojektes, der Kontinuität des wissenschaftlichen Weges.

Womit ich beim Thema dieser Sondernummer der GEOGRAPHICA HELVETICA wäre: das Wort vom «wissenschaftlichen Weg» öffnet zumindest eine Tür zum Begriff der «Methode». Dessen etymologische Verwandtschaft mit den griechischen Wortstämmen metá (hinter, hinterher, nach) und hodós (Weg, Gang) erlaubt uns, «Methode» zunächst als Weg zu beschreiben, den es zur Wissensaneignung zu beschreiten gilt. ${ }^{3} \mathrm{Als}$ «Methodologie» kann dann in der Folge diese Bestimmung erweitert werden um die im lógos bezeichnete Sprache und die ihr innewohnende Vernunft (STROHMAYER 1993). Gemäß einem solchen Verständnis wäre also «Methode» dem jeweils zu untersuchenden Sachverhalt grundsätzlich äußerlich; sie wäre kaum mehr nur als der «Pfad», der uns mehr oder weniger schnell, angemessen oder angenehm zu Einsichten verhilft. Darüber hinaus wäre eine so verstandene «Methode» stets eine «angewandte», «instrumentelle» Form der wissenschaftlichen Annäherung an Wirklichkeit. Gleichzeitig schlummert jedoch in jeder so verstandenen Methode notwendigerweise eine Vorstellung von der Sache, die es zu untersuchen gilt: ob ein «Weg», ob die «Sprache» einen Sachverhalt erreicht, ja sich einem Objekt überhaupt nur annähert, kann jeweils nur im Rückgriff auf ein im voraus postuliertes spezifisches Seinsverständnis bestimmt werden. ${ }^{4}$ «Methoden» sichern diese als selbstverständlich erscheinenden Ausgangspositionen, indem sie auf ihnen aufbauen; sie verführen geradezu zum steten ergebnisorientierten Blick nach vorn. ${ }^{5}$ Diese Art der Vorgehensweise ist nicht mit der poststrukturalistischen Kritik zu vereinbaren, ist doch ihr Interesse primär ein rückwärtsgewandtes. ${ }^{6}$ Deshalb überrascht es nicht, daß laut DERRIDA «Dekonstruktion», gar nicht als Methode zu fassen ist (1972). Aufbauend auf der Unhintergehbarkeit von «Sprache», versucht die Praxis der «Dekonstruktion», wissenschaftliche Diskurse immer wieder an ihre ureigenen Grundsetzungen zu erinnern und durch diesen Verweis aufklärend zu wirken. Als begriffsimmanente Form der Auseinandersetzung mit der Analyse von Wirklichkeit bleibt «Dekonstruktion» also gewissermaßen stationär und richtet ihr Interesse auf die jeweils vorgegebenen Raum- und Zeitkategorien. Sind diese erst einmal als idealistisch, innerlich heterogen oder auch «nur» als dualistisch konstruiert erkannt worden, öffnet sich, was im traditionell «methodisch» orientierten Denken verschlossen blieb (GASCHÉ 1986, 136-142). Konkret beinhaltet dies sowohl die Rückbesinnung auf die Kontextabhängigkeit wissenschaftlicher Erkenntnis und somit deren Historisierung als auch die Einbeziehung dessen, was im traditionellen Vollzug wissenschaftlicher Einsichtnahmen strukturell stets ausgeschlossen wurde.
Als erinnerndes Beispiel seien hier nur die dem modernen «Subjekt»-Begriff historisch anhaftenden, geschlechts-, rassen- und altersspezifischen Ausschlußmechanismen angeführt. In diesem Sinne ist also im Poststrukturalismus keine Methode im traditionellen Sinne denkbar, wohl aber das (ethische) Prinzip der Offenhaltung und Öffnung von wissenschaftlichen Diskursen oder eine permanente Form des Infragestellens. Der oft geäußerte Verdacht, daß letzteres dabei den Gewinn von wissenschaftlichen Einsichten ausschließe oder auch nur «neutral» wäre, ist dabei unbegründet: auch die poststrukturalistisch eingefärbte Form der Kritik erzeugt ja Texte und somit Texturen für Erkenntnisse anderer; nur ist bei diesen neu erzeugten Texten jener legitimatorische Bezug praktisch gelöst, der durch Methoden oft ja erst «faktisch» untermauert worden ist. Statt auf «Fakten» zu verweisen, beinhalten «Texte» nun also "Zitate», deren Verweischarakter nicht gründet, sondern weiterleitet (BENJAMIN 1983, 595).

Aus dieser Sicht muß dann auch der Unterschied zwischen «qualitativen» und «quantitativen» Methoden neu bedacht werden. Hierbei geht es nicht allein um eine oft pragmatisch motivierte Arbeitsteilung zwischen beiden Vorgehensweisen, sondern um eine progressive Kritik des Unterschieds. Grundsätzlich gilt hierbei, daß beide Vorgehensweisen die Erkennbarkeit von raumzeitlichen Strukturen und Akteuren zuerst einmal postulieren und also voraussetzen. Diese Annahme kann ihrerseits nicht methodisch einsichtig gemacht werden. Die eingangs angesprochene Verfeinerung des jeweiligen methodischen Repertoires, wie auch methodologische Genauigkeit im weitesten Sinne, erscheinen so als die Möglichkeiten, die sie zur Zeit der Aufklärung auch waren. Nicht deren Negation steht an, vielmehr sollte mittels einer radikalen Relativierung die Abtretung von wissenschaftlicher Verantwortung an Methoden gleich welchen Zuschnitts hinterfragt werden. Der «Gang», den wir auf dem Weg zum Wissen begehen müssen, ist stets ein singulärer und ist als solcher nie im voraus zu planen. ${ }^{7}$ Ein solcher «methodischer» Gang, der sich seines stets weiterleitenden Verweisungscharakters bewußt ist, negiert also nicht die methodische Vorgehensweise als solche, sondern beharrt bewußt auf deren Vielfalt. Je größer das Potential für einen bewußten Umgang mit der sprachlich vorhandenen Welt, desto vielfältiger die hierdurch abgebildeten Wirklichkeiten. In einer Welt, in der kulturelle, ökologische und soziale Reduktionen und Exklusionen zunehmend als Problem erkannt werden, wo sie vor kurzem noch als Lösung gefeiert worden sind, ist methodischer Pluralismus in seinem Verweisungscharakter schlicht und einfach ein Gebot der Stunde. Die Schwierigkeiten im Vergleich wissenschaftlicher Einsichten, die sich konsequenterweise hieraus ergeben, sind somit nicht das vermeidenswerte Produkt eines falsch verstandenen methodischen Eklektizismus, sondern reflektieren in rationaler Form eine gebotene politische Entscheidung.

Welche Folgerungen aus dem hier Gesagten zu ziehen sind, ob und inwieweit «Methode» als überholt anzuse- 
hen ist oder aber eine Neubesinnung auf methodische Vielfalt im Zuge einer Annäherung an eine poststrukturalistische Geographie angemessen ist, kann nur im Einzelfall beantwortet werden. Da mein eigenes Interesse jenen der Moderne in vielerlei Form innewohnenden räumlichen Widersprüchen gilt, will ich im Folgenden auf Beispiele aus historischen Bereichen zurückgreifen. ${ }^{8} \mathrm{Da}$ fällt zunächst auf, in welchem Umfang geographisches Denken und geographische Methoden durch die Entstehung der ihnen eigenen Selbstverständlichkeiten aus dem Umfeld des Optischen heraus geprägt sind. Aller Ideologiekritik zum Trotz überlebt die Hegemonie des Sehens bis heute und reduziert den Phänomencharakter räumlicher Sachverhalte zur einfachen, binären Logik von Anwesenheit und Abwesenheit. ${ }^{9}$ In welchem Umfang gerade geographisch relevante Sachverhalte gegen diesen elementaren Unfug rebellieren können und welcher Reichtum an Einsichten sich hinter einer solchen anfänglichen Dekonstruktion verbirgt, kann hier nur im Ansatz gezeigt werden: gerade die Bedeutung der Technik als eines der zentralen Phänomene der Neuzeit entzieht sich einer solchen simplen Form des begrifflichen Zugriffs. Dazu ein Beispiel: die Vielfalt der relevanten Wirklichkeiten, die sich hinter der begrifflichen Größe «Geld» - und hier gerade hinter dem historisch wichtigen Wandel vom Metall- zum Papiergeld - verbergen, lassen sich eben gerade nicht durch eine optisch eingeengte Logik des Vorhandenseins begreifen. Konventionell methodisches Denken, mit der ihm eigenen Trennung in Sachverhalte und Effekte, zielt an der Gleichzeitigkeit eines oft ungeordneten Widerspruchs im Materiellen vorbei (STROHMAYER 1997). Aber nicht allein das phänomenologische Primat des Optischen - und seine Umsetzung in den Bereich des Methodischen in (direkten, indirekten und archivarischen) Befragungstechniken - begrenzt die Erkenntnisfähigkeit der Geographie. Ebenso wichtig sind darüber hinaus die historisch sich durchsetzende Fortschreibung der Arbeit aus der praktischen Vernunft heraus und die hieraus sich ergebende Naturalisierung von Wissen. Hier erscheint mir die Metapher vom «Weg» - etymologisch der «Methode» implizit - als potentiell fruchtbar: Akzeptieren wir, da $\beta$ «Raum» als diachroner und synchroner Begriff nie feststellbar einen singulären Punkt darstellt, bleibt der noch bei PLATO angelegte Arbeits-, ja Raubcharakter von Wissen (HEIDEGGER 1997, 361) und die untrennbar damit verbundene politische $\mathrm{Di}$ mension methodischen Tuns unseren Diskursen gleichfalls nicht mehr fremd.

\footnotetext{
Anmerkungen

${ }^{1}$ Ich verwende im Folgenden den Begriff der «Humangeographie", in Anlehnung an den im angelsächsischen Raum vorherrschenden Sprachgebrauch, als Überbegriff für eine jede Geographie "vom Menschen». Hierdurch soll also jene
}

(wertende) Vorentscheidung zwischen "Sozialgeographie», "Anthropogeographie» oder anderen innerfachlichen Gruppierungen vermieden werden.

${ }^{2}$ Ich entlehne diesen Begriff als Übersetzung des Ausdruckes "taken-for-granted" den Arbeiten Gunnar OLSSONS (1991).

${ }^{3}$ Interessanterweise ist auch das eingangs beschriebene "periodische" Charakteristikum wissenschaftlichen Daseins demselben Wortstamm (hodós) verpflichtet!

${ }^{4}$ In diesem Rückgriff dürften die meisten Leser eine Variante des hermeneutischen Zirkelschlusses erkennen. Dessen Überwindung war und ist die (oft implizite) Aufgabe von als solchen nicht weiter zu hinterfragenden und also im wahrsten Sinne des Wortes "grundsätzlichen" fachspezifischen Grundeinheiten. Der psychologischen Dualität des Bewußtseins, seiner "Aufteilung" in bewußte und unbewußte Sphären, entspricht auf dieser Ebene der Begriff des "Textes" in den Sprachwissenschaften ebenso wie der Begriff des "Raumes" in der Geographie. Was diese Grundbegriffe eint, ist eine inhärente, strukturermöglichende Kapazität; implizit anwesend werden sie zu Bedingungen der Möglichkeit von fachspezifischem Wissen. Bevor also methodisches Denken überhaupt erst greifen kann, ist solcherart schon oft entschieden, was als "Wissen" gelten wird.

${ }^{5}$ Bezeichnend ist hier auch heute noch das von BARTELS dem methodischen Teil seiner bahnbrechenden Abhandlung vorweggestellte Zitat A. N. WHITEHEADS: “Dissonanzen sind noch unverstandene Harmonie" $(1968,160)$.

${ }^{6}$ Strenggenommen ist es nicht die Richtung des jeweiligen Blickes - auch die klassische Methodik blickt ja stets normativ auf ihren Ausgangspunkt zurück (vgl. HARD 1990) -, sondern die Ergebnisqualität solcher "Blicke", auf die unser Interesse gerichtet sein sollte.

${ }^{7}$ Hierdurch soll nun in keiner Weise die Gebundenheit wissenschaftlichen Fragens geleugnet werden. Allein durch die Anerkennung der Einbindung von Einsichten in spezifische (theoretische, ideologische oder persönliche) Fragehorizonte ergibt sich keine direkte Form der Legitimation von wissenschaftlichen Fragestellungen. Letztere bleibt also nicht allein relativ hinsichtlich der jeweils konkurrierenden Alternativen, sondern ist auch durch die ihr immanenten Vernunftdefizite relativierbar.

${ }^{8}$ Der Verweis auf historisches Arbeiten mag von manchen Lesern als Rückzieher gewertet werden, war doch streng methodisches Denken in der historischen Forschung immer schon als nur bedingt maßstabgebend anerkannt. Gleichwohl ist "historisches" Denken epistemologisch nicht verschieden von mehr gegenwartsorientierten Interessen: die sprachliche Konstitution ist sowohl der verbalen Kommunikation im Hier und Jetzt wie auch der Präsenz von Archivmaterialien eigen.

${ }^{9}$ In diesem Zusammenhang ist es interessant, einen der sicherlich bestimmenden wissenschaftstheoretischen Dispute in der Geographie, jenen zwischen idiographischen und nomothetischen Vorgehensweisen, relativiert zu sehen. Ob da nun im Einzelfall Geographie auf das Besondere oder auf das Allgemeingültige abzielt (oder abzielen soll), erscheint so als sekundäre Entscheidung, die ihrerseits auf einer (zumindest implizierten) Annahme von "Anwesenheit" oder "Abwesenheit" räumlicher Sachverhalte "gründet". So gesehen ist also die seinerzeit noch von HARD (1973) geforderte Trennung zwischen "Philosophie" und "Methodologie" in der Geographie im Einzelfall stets doch zu porös, um forschungspraktisch aufrechterhalten werden zu können. Gerade deshalb ist HARD nach wie vor zuzustimmen, wenn er schreibt, "(d)ie wichtigsten Argumente dürften disziplinpolitischer Art sein" (1973, 31; später ergänzt HARD implizit die disziplinpolitische durch die karrierepolitische Variante, vgl. 1990, 1-2). 


\section{Literatur}

BARTELS, D. (1968): Zur wissenschaftstheoretischen Grundlegung einer Geographie des Menschen (= Geographische Zeitschrift, Beihefte Heft 19), Wiesbaden.

BECKER, J. (1990): Postmoderne Modernisierung der Sozialgeographie? In: Geographische Zeitschrift 78, 1, 15-23. BENJAMIN, W. (1983): Das Passagen-Werk, 2 Bände, Frankfurt am Main.

DEAR, M. (1988): The Postmodern Challenge: Reconstructing Human Geography. In: Transactions of the Institute of British Geographers, 13, 265-274.

DEAR, M. (1994): Postmodern Human Geography. A preliminary assessment. In: Erdkunde, 48, 1, 2-13.

DERRIDA, J. (1967): De la grammatologie, Paris.

DERRIDA, J. (1972): La dissémination, Paris.

GASCHÉ, R. (1986): The Tain of the Mirror, Cambridge, Ma. HANNAH, M., und STROHMAYER, U. (1991): Ornamentalism. Geography, Language and the Autonomy of the Subject in Structuration Theory. In: Environment and Planning D: Society and Space, 9, 3.

HARD, G. (1973): Die Geographie. Eine wissenschaftstheoretische Einführung, Berlin und New York.

HARD, G. (1990): Was ist die Geographie? Re-Analyse einer Frage und ihrer möglichen Antworten. In: Geographische Zeitschrift, 78, 1, 1-14.
HARVEY, D. (1989): The Condition of Postmodernity, Oxford. HEIDEGGER, M. (1997) [1929]: Einführung in das akademische Studium. In: Gesamtausgabe Band 28, Frankfurt am Main.

LYOTARD, J-F. (1979): La condition postmoderne: rapport sur le savoir, Paris.

OLSSON, G. (1991): Lines of Power, Limits of Language, Minneapolis.

SOJA, E. (1989): Postmodern Geographies: The reassertion of space in critical social theory, London.

STROHMAYER, U. (1993): Beyond theory: the cumbersome materiality of shock. In: Environment and Planning D: Society and Space, 11, 3, 323-347.

STROHMAYER, U. (1997): Technology, modernity and the restructuring of the present in historical geographies. In: Geografiska Annaler (Series B), 73, 3, 155-169.

THRIFT, N. (1983): On the determination of social action in space and time. In: Environment and Planning D: Society and Space, 1, 1, 23-58.

WEICHHART, P. (1994): Die Welt als prästabilisierte Harmonie? Anmerkungen zu Dietrich Fliedners Versuch einer systemtheoretischen Grundlegung der Sozialgeographie. In: Geographische Zeitschrift, 82, 1, 1-24.

WERLEN, B. (1995): Sozialgeographie alltäglicher Regionalisierungen. Band 1: Zur Ontologie von Gesellschaft und Raum, Stuttgart. 